\title{
Welches Modell ist geeignet?
}

\section{Jürg Nadig \\ Dr. med., Präsident* der Schweizerischen Gesellschaft für Medizinische Onkologie, Facharzt für Medizinische Onkologie und Innere Medizin}

Der Aufbau eines nationalen Expertengremiums zu Früherkennungsfragen ist eines von 15 Projekten der Nationalen Strategie gegen Krebs (NSK) 2014-2017. Ziel des Projekts ist die Etablierung eines nationalen Expertengremiums zu Früherkennungsfragen. Unter der Koführung der Krebsliga Schweiz (KLS) und des swiss cancer screening (SCS) sowie unter Beizug der Firma advocacy ag wurde ein Diskussionspapier erarbeitet, das mögliche Modelle für ein nationales Expertengremium zu Früherkennungsfragen aufzeigt und eine erste Bewertung der Modelle vornimmt. Als Lösungsansatz stehen drei Modelle zur Diskussion (Quelle: Jahresbericht NSK 2015).

- Kommission für die Früherkennung von Krebs im Rahmen eines in der NCD-Strategie vorgesehenen Fachgremiums für Präventionsfragen («NCD-Kommission»);

- Ausserparlamentarische Kommission für die Früherkennung von Krebs («Ausserparlamentarische Kommission»);

- NPO-Expertengruppe für die Früherkennung von Krebs als Übergangslösung («NPO-Expertengruppe); Träger z.B. KLS oder oncosuisse.

Die drei obengenannten Modelle werden von der NSK zur Diskussion gestellt. Die Diskussion muss aber über diese drei Varianten hinausgehend geführt werden. Viele Prozesse und Lösungsansätze bestehen ja bereits auf nationaler und internationaler Ebene und sollten miteinbezogen werden. In der ganzen Diskussion darf der Patient zu keinem Zeitpunkt aussen vor gelassen werden.

\section{Unterschiedliche Empfehlungen}

Den Frauen zwischen 50 und 70 Jahren und ihren betreuenden Ärzten liegen in der Schweiz zum Mammographiscreening widersprechende Empfehlungen vor (vgl. Medical-board-Entscheid und Ziele der NSK). Der Wunsch, «klare Verhältnisse zu schaffen», ist deshalb verständlich. Wie findet sich aber heute eine Frau zurecht, wenn sich die Experten uneinig sind? Als salomonische Lösung sollen betroffene Frauen über Nutzen und Risiken so gut orientiert werden, dass sie gemäss ihren Präferenzen entscheiden können. Eine randomisierte Studie untersuchte, wie viele Frauen sich gemäss ihren Präferenzen für oder gegen ein Mammographiescreening entscheiden konnten [1]: Mit Informationen zur Brustkrebsmortalität und falsch positiven Diagnosen waren es $17 \%$, mit evidenzbasierten Erklärungen und quantitativen Angaben zu Überdiagnostik, Brustkrebsmortalitätsreduktion und falsch positiven Diagnosen waren es $24 \%$. 83 bzw. $76 \%$ der Frauen entschieden sich trotz diesen Informationen nicht entsprechend ihren Wünschen und Wertvorstellungen. Vom Ideal, aufgrund der Evidenz gemäss eigener Präferenzen mitentscheiden zu können, sind wir noch weit entfernt.

\section{Kommunikation von Risiko}

Früherkennungsfragen gemeinsam und zentral ist auf allen Entscheidungsstufen (Patient/Arzt, Experte/Politiker, Bevölkerung/Medien) die Kommunikation von Risikoverminderung aufgrund evidenzbasierter Informationen. Entscheidungsträgern, Ärzten und Betroffenen gemeinsam ist aber auch, dass sie weder in der Erarbeitung der Evidenz noch in der Kommunikation von Risiken geschult sind. Damit werden sie leicht zum Spielball von Interessengruppen, die die vorhandene Evidenz zu ihren Gunsten interpretieren. Würden europaweit alle Frauen zwischen 50 und 70 zehn Mal zu Schweizer Preisen (100 Franken/Screening) mammographiert, bringt dies bei einer 100\%-Beteiligung 370 Milliarden Franken pro Generation in den medizinisch industriellen Komplex. Dies mag mit ein Grund sein, dass diese Frage so umstritten ist. 


\section{Kostspielige HTA-Prozesse}

Health Technology Assessment(HTA)-Prozesse sind aufwendig und kostspielig. In einem ersten Schritt sammelt, sichtet und bewertet ein HTA-Expertengremium (mit Einbezug der betroffenen medizinischen Fachgesellschaften) die vorhandenen Daten und bereitet sie zu einem Bericht auf, der für die Adressaten so verständlich abgefasst sein soll, dass sie Entscheide fällen können. Dabei kann es sinnvoll sein, dass in einem ersten Schritt Fachleute das Fachwissen sammeln, während in einem zweiten Schritt ein anders zusammengesetztes Gremium jeweils unter Berücksichtigung sozialer, rechtlicher und ethischer Aspekte die Empfehlungen ausarbeitet, die den Entscheidungsträgern (auf politischer oder individueller Ebene) zur Verfügung stehen.

\section{Worauf können sich Ärzte stützen, wenn sie in der Praxis Frauen beraten?}

Je komplexer ein Problem ist, umso mehr wünschen wir uns einfache klare Antworten. Gleichzeitig fordern wir aber, in den Entscheidungsprozess miteinbezogen zu werden (Shared decision making [2]). Wie können wir in der Praxis mit dieser Schwierigkeit umgehen?
Bezüglich der Evidenz können wir uns bei der Beratung zu Screeningfragen primär auf die Cochrane Database of Systematic Reviews [3] stützen. Diese unabhängige Institution hat eine hohe Reputation (vergleichbar dem Intergovernmental Panel on Climate Change, kurz IPCC, das wissenschaftliche Fakten zur Klimaveränderung zusammenstellt). Diese Datenbasis ist dank der SAMW nun jedem Schweizer Arzt zugänglich. Zum eigenen Verständnis und zur verständlichen Vermittlung des Nutzens von Früherkennungsmassnahmen an die Ratsuchenden eignet sich die Faktenbox des Harding-Zentrums für Risikokompetenz [4]. So erfolgt die Sichtung und Aufarbeitung der Evidenz durch kompetente, anerkannte und unabhängige Fachleute. Diese stellen uns sowohl die notwendigen Unterlagen für die Risikobeurteilung als auch die Hilfsmittel für die Risikokommunikation mit den betroffenen Frauen und ihren Angehörigen zur Verfügung. Beide Quellen sind international anerkannt.

\section{Welche Aufgabe käme dem Experten- gremium für Früherkennung der NSK zu?}

Die für die Beratung der betroffenen Frauen zur Verfügung stehenden Unterlagen (Cochrane Database of Systematic Reviews und die Faktenboxen des Harding

\section{Brustkrebs-Früherkennung}

durch Mammographie-Screening

Zahlen für Frauen ab 50 Jahre, die 10 Jahre oder länger am Screening teilgenommen haben

1000 Frauen ohne Screening:

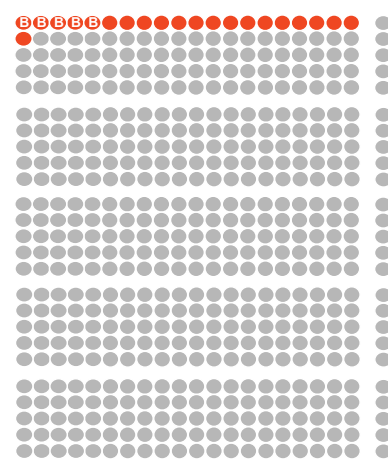
ᄂ

B Frauen, die an Brustkrebs starben:

- Frauen, die insgesamt an Krebs starben:

- Frauen, die nach einer Biopsie erfuhren, dass inr Testergebnis falsch positiv war:

- Frauen, die unnötig mit Brustkrebs diagnostiziert und behandelt wurden:

übrige Frauen:
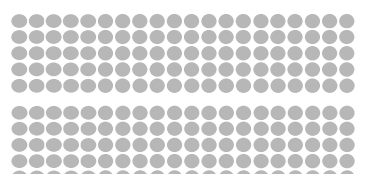

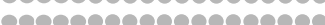

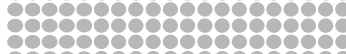

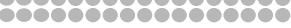

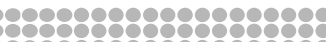


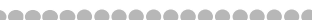

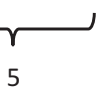

21

$-$

$-$

$-$

979
1000 Frauen mit Screening:

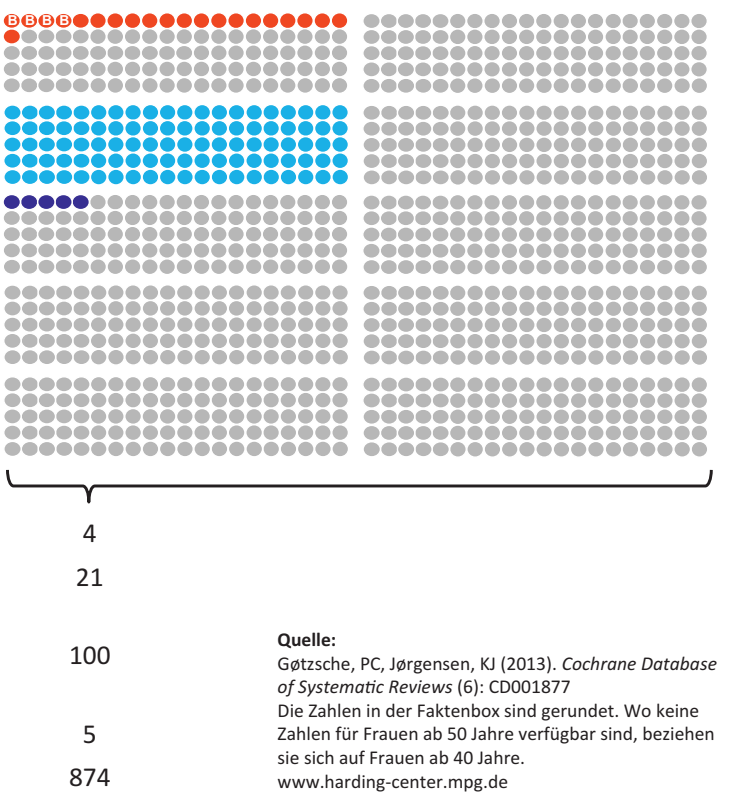


Zentrums für Risikokompetenz) sind öffentlich zugänglich und stehen jedermann zur Verfügung. Das Expertengremium im Projekt 2.3. der NSK muss diese Arbeit nicht nochmals durchführen. Ziel der NSK ist ja, bereits vorhandenes Wissen zu bündeln. Die Aufgabe «Evidenz aufarbeiten» entfällt somit. Das Expertengremium ist deshalb kein IPCC. Die Schlüsse, die einzelne Akteure aus der zur Verfügung stehenden Evidenz ziehen, können je nach Interessenlage natürlich diametral verschieden sein. Ein Hersteller von Mammographiegeräten mag Wert darauf legen, dass die Sterberate an Brustkrebs durch Screeningmammographie sinkt. Der operativ tätige Gynäkologe oder ein Spital wird für die Mammographie votieren, weil damit die Zahl der Eingriffe steigt. Fundraisingorganisationen, die an einem guten Image bei Frauen interessiert sind, damit die Spendengelder üppig fliessen, werden medienwirksam dafür kämpfen, dass den Frauen die Mammographie nicht vorenthalten wird. erfahren.» Das ist der Qualitätsstandard, an dem alle Projekte der NSK zu messen sind. Mit dieser Aufgabendefinition wird dieses Expertengremium weder zu einem HTA-Organ wie der IPCC, noch verkommt es zu einem Politgremium, in dem die verschiedenen Stakeholder versuchen, ihre Partikularinteressen durchzusetzen. Vielmehr wird das Expertengremium zu einem Schiedsgericht. Es hat lediglich aufgrund der in den Faktenboxen aufbereiteten Evidenz zu beurteilen, ob mit einer Screeningmassnahme die in der NSK formulierten Ziele erreicht werden oder nicht. Daraus lässt sich auch die Zusammensetzung des Expertengremiums ableiten: Am besten entscheiden drei erfahrene Richter, ob die NSK-Ziele erreicht werden.

$\mathrm{Zu}$ fragen ist also, welche der Ziele der NSK aufgrund der in der Faktenbox zusammengestellten Evidenz beispielsweise durch die Screeningmammographie erreicht werden. Eine mögliche Antwort liefert folgende Tabelle:

\begin{tabular}{|c|c|c|}
\hline 1. Sterben weniger Menschen an Krebs? & Nein & $\begin{array}{l}\text { Mit und ohne Screeningmammographie ster- } \\
\text { ben } 21 \text { von } 1000 \text { Frauen an Krebs }\end{array}$ \\
\hline 2. Erkranken weniger Menschen an Krebs? & Nein, es erkranken mehr & $\begin{array}{l}\text { Dank der Screeningmammographie wurden } \\
5 \text { Frauen wegen eines Brustkrebses behandelt, } \\
\text { an dem sie nie manifest erkrankt wären }\end{array}$ \\
\hline 4. Werden mehr Menschen von Krebs geheilt? & $?$ & $\begin{array}{l}\text { Es werden } 5 \text { Frauen von einem Brustkrebs } \\
\text { "geheilt», an dem sie nie manifest erkrankt } \\
\text { wären, was kaum einer Heilung gleichgesetzt } \\
\text { werden kann }\end{array}$ \\
\hline
\end{tabular}

\section{Weder IPCC noch Klimakonferenz}

Soll dieses Expertengremium für Früherkennungsfragen nun, wie die Klimakonferenz, politisch die verschiedenen Interessen austarieren und in einem «ausgewogenen evidenzbasierten» Bericht zusammenfassen und so Empfehlungen für Ja/Nein-Entscheide zu liefern? Wohl kaum. Es handelt sich ja um ein Expertengremium im Rahmen der NSK. Deshalb soll seine Aufgabe darin bestehen, anhand der vorhandenen Faktenboxen zu beurteilen, ob mit einer Screeningmassnahme die Ziele der NSK erreicht werden oder nicht. Diese Ziele sind in der Vision der NSK 2014-2017 klar festgehalten: «Die Nationale Strategie gegen Krebs strebt eine Schweiz an, in der weniger Menschen an Krebs erkranken, weniger Menschen an den Folgen von Krebs leiden und sterben, mehr Menschen von Krebs geheilt werden und Betroffene und ihre Angehörigen in allen Phasen der Krankheit aktiv miteinbezogen werden und die nötige Zuwendung und Hilfe
Unabhängig vom Entscheid des Schiedsgerichts steht es in einem nächsten Schritt den Entscheidungsträgern frei, Früherkennungsmassnahmen aus politischen Gründen oder zum Erhalt der Vollbeschäftigung ein- oder weiterzuführen, auch wenn sie, wie möglicherweise beim Mammkarzinomscreening, den Visionen der NSK allenfalls zuwiderlaufen. Die Eidgenössische Leistungskommission (ELK) kann den Entscheid des Expertengremiums in ihre Zulassungsentscheide einbeziehen. Ist eine Massnahme unwirksam oder sogar schädlich bezüglich der Vision der NSK, müssen Zweckmässigkeit und Wirtschaftlichkeit gar nicht erst geprüft werden. In Übereinstimmung mit dem Choosing-wisely-Konzept könnte die ELK die Leistung auch aus dem Pflichtleistungskatalog streichen.

\section{Literatur}

1 Lancet. 2015;385:1642.

2 http://www.fmh.ch/files/pdf16/Grundlagenpapier_SDM.pdf

3 http://community.cochrane.org/cochrane-reviews

4 https://www.harding-center.mpg.de/de/gesundheitsinformationen/faktenboxen/ 